\title{
Stress Distribution in Materials
}

\author{
A Review of Various Methods Suggested for the Solution of Important Engineering Problems
}

\author{
By Prof. E. G. Coker, M.A., D.Sc., M.Inst.C.E.
}

\begin{abstract}
The subject of stress distribution in materials, which I have chosen for this address, is not one which an engineer can claim as his peculiar province, for it ha been and still is a fruitful field of investigation for the mathematician, the physicist and the geologist, and has always been so since the commencement of scientific
\end{abstract} inquiry.

The development of architecture from the earliest dwellings of savage races to the great temples of Egypt and Greece, the bridges and aqueducts of the Romans, and the mediæval buildings of Europe, all bear witnes to the accumulation of practical knowledge of the properties of materials and of the stress distribution in struc tures, which we cannot fail to admire, although we know far too little of the way in which these ancient structure were planned and constructed. The magnificent arched and domed buildings of the Roman period, and the stately cathedrals of later times with their wealth of architectural form-tower and spire, flying buttress and vaulting - al show how considerable was the practical knowledge of stress distribution possessed by the master builders who planned and carried out these great structures. We, who inherit these buildings as a precious legacy of bygone ages, have at our command far greater resources in the accumulated knowledge of centuries of scientific discover and invention, and can build more complex structuresgreat bridges of steel, towering frameworks covered by a thin veneer of masonry, and floating arsenals of the most bewildering intricacy. All these we can show to our credit as the result of the steady increase of scientific knowledge applied to practical ends, but, even now, knowledge of the stresses which come upon these comknowledge of the stresses which come upon these com-
plex structures and machines is relatively small. Scientific investigations of engineering problems of stress still lag behind constructive ability, and defective knowledge is obscured more or less by approximate theories and buttressed by factors of safety, which serve in one instance perhaps, but show in others that they have merely given a sense of fancied security with no real basis, and are more properly factors of ignorance, to be discarded are more properly factors of ignorance, to be discarded
at the earliest moment. Who, for example, can say with certainty what is the stress distribution throughout the certainty what is the stress distribution throughout the
compression members of a great bridge, built up of complicated steel shapes and plates, united by stiffening angles, gusset plates, and innumerable rivets? There is probably good reason for the belief that a great strut is relatively weaker than a small one, when both are designed according to the same approximate formulm now used in current practice, and engineers are unwilling to take the responsibility for such members in a great structure, without providing a very ample margin of safety to cover the contingencies arising from lack of precise knowledge of the strength of these members. So numerous are the problems which arise in the design and construction of machines and structures, that it is perhaps not unprofitable to devote a short hour to the consideration of some of the available means which an engineer can use as a guide for his applications of science to construction, since of whatever kind are the professional activities he pursues, his place in the scheme of affair mainly depends on his ability to make machines and structures for directing and modifying natural sources of power in known ways, or applying them to new pur-
poses as scientific discoveries advance the boundaries of knowledge.

It is of some help to our appreciation of the achievements of the great constructors of past ages, if we remember that they probably all held the erroneous view that materials of construction are perfectly rigid bodies,
and, indeed, we know that as late as 1638 Galileo Galilei and, indeed, we know that as late as 1638 Galileo Galilei was of that opinion, and that he came to an entirely
wrong conclusion as regards the stress distribution in wrong conclusion

From the standpoint of the engineer, nothing is of more practical importance than the great discoveries of Hooke and Young, that bodies like metal, wood, and between stress and strain. It is probably within the mark to say that nine tenths of all the experimental inmark to say that nine tenths of all the experimental in veen entirely based on the fundamental principles which they enunciated, and new uses are continually arising. The recent application of the steam turbine to the propulsion of ships produced a profound change in marineengine practice, and incidentally involved an entire reconstruction of methods for obtaining the horse-power developed, which had been gradually perfected from the * An address to the Engineering Section of the British Associa-
tion for the Advancement of Science by Prof. E. G. Coker, M.A. tion for the Advancement of Science by Prof.
D.Sc., M.Inst.C.E., President of the Section. time of Watt, but were absolutely useless for the new system of propulsion. Hooke's discovery of the essential pringiness of metals enabled engineers quickly to devise new instruments capable of accurately measuring the infinitesimal angular distortions of propeller shafts, and from these to determine the horse-power transmitted by the aid of an appropriate modulus.

The construction of tall buildings affords another example where advantage has been taken to determine the loads upon columns by measuring the minute diminutions of length as the structure proceeds, thereby affording a valuable chesk upon the calculations for these members, and a reliable indication of the pressures supported by the foundations.

The distribution of stress in buildings constructed of composite materials like concrete reinforced with stee has also been examined by similar methods, and much data for guidance in future constructional work has been obtained, especially in the United States of America.

The still more difficult problems involved in the determination of the stresses in joints and fastenings of commination of the stresses in joints and fastenings of com-
plicated structures have often been investigated by purely mechanical measurements of strain, and the experimental investigations of Professors Barraclough and Gibson and their pupils upon the distribution of stress due to riveted joints and curved plates of boiler shells afford a notable example of the successful application of the measurement of small strains to a stress problem of great complexity.

That "science is measurement" is here sufficiently obvious, and it seems only due to the memory of that great engineer, Sir. Joseph Whitworth, to refer to his great mechanical achievements of a true plane and well-nigh perfect screw, which enabled him to measure changes of one millionth of an inch, and thereby gave experimental investigations of strains a new impetus, which is reflected in subsequent work on the subject. Nor must we forget the no less important exposition, by Kelvin and Tait, of the scientific principles of instrument construction which have done so much for the design of instruments for the precise measurement of strains.

Mechanicalmeasurements cannot, however, completely satist, all our modern requirements, since they are essentially average values, and fail to accommodate themselves to many of the problems which press for solution.

In the quest for exact experimental knowledge, the measurement of stress at a point becomes of paramount importance, and we may, therefore, inquire what further disposal for the determination of stress distribution in materials.

It is well known that many materials when tested to destruction show a considerable rise of temperature at the place of fracture, especially in very ductile materials;
but Weber was the first to discover that a metal wire but Weber was the first to discover that a metal wire when stretched within the elastic limit is cooled by the action of the load, and this result was deducted later from the laws of thermo-elastic behavior of materials by Lord Kelvin, who showed that tension and compression loads produce opposite effects, and that materials which have the property of contracting with rise of temperature show thermal effects of the reverse kind. Although the changes of temperature produced by stress are small
within the elastic range--less than $1 \mathrm{deg}$. Cent. for most within the elastic range--less than $1 \mathrm{deg}$. Cent. for most
materials-yet their effect upon a thermo-couple is readily measurable if the equilibrating effects of surrounding bodies are neutralized or allowed for, so that stress distribution can be determined by thermal measurements at a point. The correction for such disturbing causes is usually an important factor, and is generally so large that experimental work is more suitable for the laboratory than the workshop; but if all necessary precautions are taken a linear relation of stress to strain can be shown to hold up to the elastic limit of the material, while above this point the break-down of the structure causes a rise of temperature of so marked a character that it has been utilized by several investigators as an indication of the yield point.

Experiments, upon members subjected to tension, compression, and bending, show that thermal phenomena afford trustworthy indications of the stress in materials so diverse as a rolled-steel section, a block of cement, and beams of stone and slate. Although no attempt appears to have been made to investigate stress distributions of any great complexity, it seems not unlikely that thermal methods of investigation will ultimately prove of con siderable value.

The transparency of metals to Röntgen rays is another phenomenon which has often been suggested as likely to be of service for work an stress distribution in materials, and Mr. Howgrave Graham and I have examined a number of rolled metals under stress up to the breaking point, without however, discovering any change in the appearance of the material as seen on a fluorescent screen. Although our experiments showed no preceptible change, it is, of course, not impossible that an effect may have escaped our notice.

Another and still more fascinating field of research on stress distribution is afforded by the doubly retractive properties of transparent bodies under stress, a discovery made by Sir David Brewster almost exactly one hundred years ago, and but rarely made use of since by engineers, although Brewster himself immediately saw its value for experimental purposes, and suggested that models of arches might be made of glass, and the effects of stresses due to loading rendered visible in polarized light. Brewster carried his investigations further, by the invention of a "chromatic teinometer" for investigating the nature of strains, and consisting of plates or bars of glass subjected to flexure in definite ways for comparison with the body under stress.

At a much later date (1841), Neumann developed an elaborate theory for the analysis of strain in transparent bodies due to load, unequal temperature, and set, while still later the youthful genius of Clerk-Maxwell supplied an algebraic solution for the stress distribution in any plate subjected to stresses in its own plane.

The application of optical investigation to the determination of stress distribution in engineering structures and machines has, however, been hindered by causes which, although apparently insignificant, have been very real obstacles, and among these was the absence of a transparent material which could be fashioned into shapes suitable for investigating technical problems. It is not an easy matter, for example, to construct a glass model of a bridge free from internal stress, in the manner suggested by Brewster; and, moreover, glass is extremely fragile under load, especially in cases where the stress distribution in it varies very much, while the cost of construction is very great. Happily there is now no necessity to employ glass for experimental investigation on engineering problems, since modern chemistry has supplied artificial bodies, such as the nitro-cellulose compounds which have optical properties very little inferior to glass, are able to bear great stresses without injury, and also are capable of being fashioned with the ease and certainty of a wooden model. Photographic processes are also able to reproduce the brilliant color effects caused by stress in transparent materials, so that permanent records can now be made for future reference.

The construction of polariscopes for examining models on a large scale is very essential for technical research, and the great scarcity of Iceland spar of sufficient purity and size for use as Nicol's prisms has caused much attention to be paid to the construction of apparatus for producing plane polarized light by the aid of sheets of glass. Fortunately this presents little difficulty, and although the light is not nearly so well polarized as that obtained from a Nicol's prism it is sufficiently so for the purpose. Large quarter-wave plates of mica have also been constructed by my colleague, Prof. Silvanus Thompson, F.R.S., for obtaining circularly polarized light, and thes have proved sufficiently exact and exceedingly useful for large models.

It is of importance to show that the stress distribution revealed by a polarized beam of light passing through an elastic transparent material in no ways differs from that obtained by other means, and evidence is available in modern researches, especially by Filon, that the experimental results obtained with glass agree with those of the theory of elasticity, while a satisfactory agreement of a similar kind has also been obtained with nitrocellulose compounds, although not in so complete and direct a manner. Such an agreement may be expected on theoretical grounds, since the values of the elastio constants do not affect the fundamental equations for stresses in a plane, and although for three-dimensional stress the effect of the stretch-squeeze ratio causes some difference, yet this is usually negligible.

Most of the physical constants of glass have been determined with very considerable accuracy, but other transparent substances have so far received little attention, and their optical constants are not well known. The stress-strain relations of glass and nitro-cellulose have been determined with considerable accuracy, and a useful idea of their relation to metals may be gained from the values of the stretch-modulus, $\mathrm{E}$, and the stretch-squeeze ratio, $\sigma$.

The accompanying table shows some average values 
that the stretch-squeeze ratios of cast iron and plateglass are very similar, while the values of the stretch modulus are nearly as three to two. These two materials also possess other like characteristics: they are both very brittle, and possess well-developed crystalline structure so that we may expeet the properties of east iron unde stress to be very faithfully followed by plate-glass.

\begin{tabular}{|c|c|c|}
\hline Material. & $\mathrm{E}$ & $\sigma$ \\
\hline Steel........ & $30,000,000$ & 0.25 \\
\hline Wrought iron. & $28,000,000$ & 0.28 \\
\hline Cast ir & $15,000,000$ & 0.25 \\
\hline Plate-glass & $10,500,000$ & 0.23 \\
\hline Nitro-cellulos & 260,000 t• 300,000 & 040 \\
\hline
\end{tabular}

The high values of the stretch modulus for steel and wrought iron are not, apparently, approached by any transparent material having similar ductile properties, but although nitro-cellulose has a stretch modulus of rather less than one hundredth that of steel, its stressstrain properties are not unlike. In some recent experiments with a miniature testing machine fitted with an arrangement for recording the stress-strain relations of xylonite throughout the whole range of stress up to frac ture, the main characteristics of steel appear on a ver much reduced scale; and give additional confidence that
the results of optical experiments on this material are applicable to metal structures.

The complete analysis of stress distribution in a plate is not, however, a simple matter, and the analysis of Clerk-Maxwell was intended to provide a solution based on the properties of the isochromatic and isoclinic line coupled with the law that the optical effect is proportiona to the difference of the principal stresses at a point, an to the thickness of the plate.

A principal stress perpendicular to the bounding planes is assumed to have no optical effect; but since many cases ponents, it seemed desirable to examine such a case exporimentally.

Some observations on circular plates clamped at the edges and uniformly loaded over one face, showed that the bending stresses produced in the plate caused ver little optical effect, since the tension and compression stresses neutralized one another, while the shear effect also appeared to be practically negligible. The only remaining stresses of importance were those caused by the clamping plates at the boundary, which produced radial and circumferential stresses having circular symmetry, and as the optical effects of these latter disappeared at a small distance from the edge, a field of view was obtained in which the optical effects of load applied perpendicularly to the plate were quite small, even when the interna stresses were very great.

Two circular plates clamped together to enclose a space between them may therefore be used as windows for observing the effect of a uniform pressure upon a transparent specimen, which latter may be a plate with its faces parallel to the end plates closing the chamber. If cubical compression is applied by a fluid, the principal cubical compression is applied by a fluid, the principal
stresses in the plane of the plate produce opposing optical effects, and any remaining effect is due to perpendicula pressures on the faces. The arrangement of experimental apparatus, therefoie, took the form of a pair of trans parent windows separated by an annular disk, and firmly clamped together by collars. The central chamber so formed was subjected to pressure of air, or other fluid, up to about one thousand pounds per square inch, and afterward the specimen was introduced and the same pressure ward the specimen was introduced and the same pressure Finally, the specimen was set in the field of view outside the chamber, and pressure again applied by the fluid, but still no change was apparent. In all three cases the optical effects produced were small, and practically alike so that the experimental evidence appears to warrant the conclusion that a principal stress in the direction of an incident beam of polarized light has no optical effect neglected.

That the retardation between the ordinary and extraordinary rays is proportional to the stress difference perpendicular to the incident beam within the elastic limi of the material may, therefore, be taken as reasonably accurate, although future research may show that it is only an approximation, or even that it is more accurate to commence from a fundamental strain equation; but according to present knowledge there appears to be no warrant for such a procedure.

A more pressing difficulty arises with regard to the optical constant connecting the wave-length retardation with the stress difference. The recent researches of Filon on glass show that the value of this constant is curiously dependent on the previous history of the material, especially as regards its heat treatment. Until further knowledge is gained on this matter it appears to be necessary to guard against errors in stress measurement from this cause by a careful selection and treatment of the material used, since for other artificial bodies we may
find that the variation in the constant is not less in mag- nitude, and is at least as complex as in glass. In some nstances the stress optical coefficient may be dispensed with, and Filon has shown, in eases where a theory of stress distribution has been worked out and it is desired to compare it with the results of optical measurements, that the isoclinic lines offer many advantages, since they are independent of photo-elastic constants, and

The experimental analysis of stress distribution in a body depends on the possibility of finding the magnitudes and directions of the principal stresses at every point, and in practice it is found the simplest plan to determine and in practice it is found the simplest plan to determine obtained in plane polarized light, and to measure the stress difference by comparison with a wave-length standard, such as a Babinet compensator, or by comparison with a simple tension member set along one of the lines of principal stress, and loaded until the total effect produced is a dark field denoting a zero value. The difference of the principal stresses is then measured in terms of a of the principal stresses is then measured in terms of a
simple tension. This alone is insufficient. to determine the distribution, unless one of the principal stresses is zero, and, in general another independent measure must be obtained. This is very conveniently supplied by the change in the lateral dimensions of the plate under stress, since this change may be taken, in the absence of a third principal stress, as proportional to the generalized sum of the principal stresses throughout the thickness.

The determination of the lateral strains in a comparatively thin plate, forming part of a model of a machine or structure, necessitates measurements of extremely minute linear quantities. If, for example, a plate of xylonite is taken, of the maximum thickmess obtainable for optical work, a simple calculation shows that these strains must be measured to an accuracy of one or two millionths of an inch. Several instruments have been designed and constructed for this purpose, to fulfil conditions which appear to be essential for successful use. It is necessary to avoid all chance of injury to the surface of a transparent material, so that the measuring points of an instrument can only be pressed lightly against the surface, and the weight must, therefore, be supported independently of the model. In instruments so far constructed, the measuring mechanism is carried on a $\mathrm{U}$ shaped frame, for convenience of movement from point to point of the specimen. One measuring needle is secured and operated by a calibrating screw, and the other is free to move a multiplying lever system, and thereby tilt a mirror to give an angular deflection, which latter is calibrated by reference to the standard screw when the instrument has been finally secured in place. In recent work the labor of accurately setting the instrument in a number of different positions has proved so great, that my assistant, Mr. F. H. Withycombe, has designed a useful adjunct in the form of a mechanical slide-rest, to effect the required changes easily and expeditiously. In one arrangement, a bracket carries the measuring instrument on a three-point support, and movement is effected by slides arranged to give displacements along three axes at right angles, and their amounts are measured by micrometer screws to an accuracy of rather less than one thousandth of an inch.

These methods of stress determination avoid the diffculties of the Clerk-Maxwell analysis, which necessitates the determination of the equations to both families of isochromatic and isoclinic bands, usually a mathematical problem of considerable complexity. In some simple the method of lateral measurements for determining the sum of the principal stresses, by comparing the calculated stresses with the experimental values obtained in a plate of transparent material. We have lately carried these experiments a stage further, and have shown that the measured sums of the principal stresses in steel agree with the calculated values. This experimental solution, in fact, gives the stress at a point in a plate, if the conditions are those assumed by the mathematical case of plate where generalized equations of stress apply.

It is at once obvious, if the utility of experiments on models of this kind is admitted, that experimental evidence is available on a variety of practical engineering problems covering a very wide field of practice, not merely qualitative, but quantitative, and approximating to the needs of the physicist and mathematician, and well within the known variations of the materials

During the last few years much attention has been paid to the determination of the stresses in structural elements of primary importance, but only a small number of cases have been examined, since even the simplest problems have proved somewhat difficult, and much time and labor have been spent in perfecting optical and mechanical appliances to suit the special conditions required for investigations on transparent models. A simple example of a case easily examined and of practical importance is that of a tension member subjected to an eccentric load. The optical effects here show a linear distribution of stress due to the combination of direct pull and bending, while the neutral axis moves toward the tension side as the stress increases. Not only can these effects be measured, but if the specimen begins to fail some indication is obtained of the way in which the stress distribution is changed to meet the new conditions, and there is found a tendency to an equalization of the maximum stress at the boundary, although at elastic limit is largely conjectural.

A case like that of a very short member subjected to direct compression is also not without interest, partly because it reveals unexpected difficulties. In the first place it is not easy to apply a pure compression stress, and if the surfaces in contact are not of the same materials it appears to be practically impossible, since the lateral changes are unlike, and shear stress is therefore produced at the plane of the surfaces in contact. In a short member this shear has a very important influence, and by interposing a thin layer of a material, such as india-rubber, between the pressure plates and the short transparentblock, the artificial shear effect produced by the indiarubber is easily shown to influence the distribution throughout, and to increase the stress in a very marked way. Experiments on transparent materials show that the increase of stress may be twenty per cent or even more. Such an effect is known to take place when cubes of stone are crushed between lead plates, and optical investigations on models have enabled a quantitative measure of the effect to be ascertained in this and other cases, thereby confirming the theoretical investigations of Filon on the distribution of stress in such members under various practical systems of loading.

The local effects produced near the points of application of a load are usually of considerable importance, and their influence on the stress distribution in beams has been examined by Carus-Wilson.

The stress effects produced by discontinuities in materials are also of considerable interest, and the cases arising from the necessities of construction are infinite in their variety.

The practical importance of an accurate knowledge of the change in stress distribution produced by changes of section in a member is so thoroughly appreciated that it needs no insistence, and it has received much attention from a mathematical point of view. Thus the local effect of a spherical cavity in a member subjected to uniform tension or compression load has been shown by Love to double the intensity very nearly, while Kirsch has shown that a small eylindrical hole in a tension member trebles the stress intensity. If the hole is elliptical the increase of stress may be still greater, and Inglis has shown, among other interesting cases, that if the minor axis of the ellipse is parallel to the direction of the applied load in a tension member, the stress intensity is increased by an amount measured by twice the ratio of the axis of the ellipse.

A crack, considered as the limiting case of an elliptical hole, is thus seen to give extremely great stresses at the crack.

Optical experiments afford an independent means of examining the alterations of stress intensity produced by discontinuities, and the results are found to agree remarkably well with those obtained from the theory of drical hole in a plate has been found to be almost exactly three times the stress in the full plate, and the effects of holes comparable with the width of the tension member have also been examined in some detail.

In the case of a rivet just filling the hole and exerting no tangential effect at the boundary, there is a lessened tension stress across the minimum section at the boundary hole, accompanied by a marked radial tension. These effects have been recently confirmed in a mathematical discussion by Suyehiro. Other cases give satisfactory agreement with calculation, and we may therefore feel some confidence that experimental investigation will prove useful in some of the very complicated cases arising out of engineering practice where analysis is difficult, if not impossible.

The effects of overstress in materials may also be examined by optical means, and although the laws relating to stress distribution in overstressed transparent material are not known, the general effects observed in simple cases are fairly evident. If, for example, a tension member of glass is stressed, there is no ductile yielding of the material, and the stress will therefore rise very rapidly at the boundary of a small hole, and fracture will therefore occur with a moderate load. If, however, a ductile transparent material is employed, and the material hows signs of failure at the hole, the breakdown of the structure spreads outward as the load is increased, until we may have a condition in which, within the elastic limit, the curve of stress intensity at the minimum section accords with calculation, but at the overstressed part the stress tends to equalize, and the curve of intensity tends to become horizontal near the hole. The mean value of this part of the stress distribution may be invalue of this par measured values below the region of failure; but the true 
distribution of the overstress has not been accurately determined, so that the shape of this peak is largely conjectural.

The effects of groups of rivets such as occur in bridges, boilers, and structural members of all kinds, afford ample scope for further inquiry; but before more exact knowledge can be gained of the condition of stress in a com plicated riveted joint it appears necessary to examin thoroughly the very simple cases.

Other interesting cases of discontinuity in structure are afforded by the engine hatchways, gun-turrets, funnel openings, and the like, in ships' decks, and some progress in this direction has been made by experiments on model decks, subjected to loads like those produced when decks, subjected to loads like those produ.
vessel meets the waves due to a head sea.

Even if the utility of transparent models is left out of account, it is generally acknowledged that many engineering problems are often simplified by the use of models of machines and structures on a small scale, where circumstances forbid experimental examination of the actual work. No defense of their use is, I think, necessary, since the employment of models is a characteristic feature of British methods, not limited to engineers. Kelvin did not disdain their use, and his successors, who have done so much to advance knowledge of the ether have done so much to advance knowledge of the ether
and the atomic dust, have freely employed their great and the atomic dust, have freely employed their great ingenuity in the construction of mechanical models an diagrams to explain their views, as in the Lodge cog wheel diagrams of the ether, the planetary systems of of elements by Soddy.

Engineers have not the same great difficulties which confront those who are advancing the boundaries of pure science; their models are very much what they please to make them; but, even then, problems arise which ar sufficiently difficult to tax all the resources of applied science. The behavior of models considered as similar structures is, therefore, a subject which engineers are bound to investigate in order to determine the effects of fixed and moving loads, the action of wind, the pressure and frictional effects of steam and other fluids, and many ather problems.

In the majority of cases the simplest and the most direct method is the experimental study of a model, from which to obtain the data required for calculating effect on a full-sized structure, and hence the laws of similarity have received a very close scrutiny.

Although most valuable information can be obtained from models, their usefulness is clearly limited. The effects of the dead weight of a structure are proportional to the cube of the linear dimensions, and are, therefore, not usually measurable on a model except in exceptional circumstances, as, for instance, where elastic jellies are circumstances, as, for instance, where elastic jellies are
employed, as in the well-known investigations of Pearson on the stress distribution in reservoir dams. Nor are questions of stability easy to solve, since the forces producing instability are proportional to the size of the model. On the other hand, stress effects due to applications of load may be measured by the strains produced in a model of the same material, if the loads are proportional to the squares of the linear dimensions. The effect of applied load are studied even better in a model conof applied load are studied even better in a model constructed of transparent material, since the variation of
stress from point to point can be studied with much greater ease and certainty.

As detailed models of this latter kind present some variations from the usual laws of similarity, it may be of interest to indicate their nature. Questions of deformation clearly involve the elastic constants of the transparent material and their relation to those of the proposed structure, while stress distribution in the solid is influenced by the value of Poisson's ratio. This latter effect is quite small for glass, but may become appreciable with other substance. It is negligible in a model of any material which approximates to a thin plate stressed by forces in its own plane.

The optical effects for any given load are, moreover independent of the thickness of the material, and depend upon the stress difference, so that color effects are obtained which may be regarded as pictures of shear stress throughout the model. Modern researches on ductile throughout the model. Modern researches on ductile
materials like structural steel indicate that such materials materials like structural steel indicate that such materials
fail at some limiting value of shearing stress, and since the places where these limiting values are reached in the model are visible to the eye, the weak places in the design of a structure can be ascertained and a faulty design corrected by purely experimental means.

In this connection it is of interest to mention that M. Mesnager, the chief engineer of bridges and roads to the French Government, has recently constructed an elaborate model in glass of a design for an arched bridge of about 310 feet span. This investigation was considered advisable for a work of this magnitude constructed of reinforced concrete, in order to check the calculations, especially of maximum stresses in the arched ribs, which latter were assumed to be fixed at the ends.

The effects of reinforcements were allowed for by determining equivalent sections of glass for the members of the model. Many difficulties had to be overcome in the production of a model free from optical defects, but these were all successfully surmounted. The stresses in the model were determined by aid of a Babinet compensator, and formed a valuable check upon the calculations for a structure of this great magnitude and somewhat unusual design.

In this brief and incomplete account of a small branch of applied science relating to engineering the fundamental importance of discoveries in pure science is manifest.

\section{A Homemade Fly Trap}

A Recent issue of the Weekly News Letter to Crop Correspondents, issued by the Department of Agriculture, gives a description of a cheap and easily constructed fly trap that was sent to the Department by a farm demonstration agent in South Carolina, where

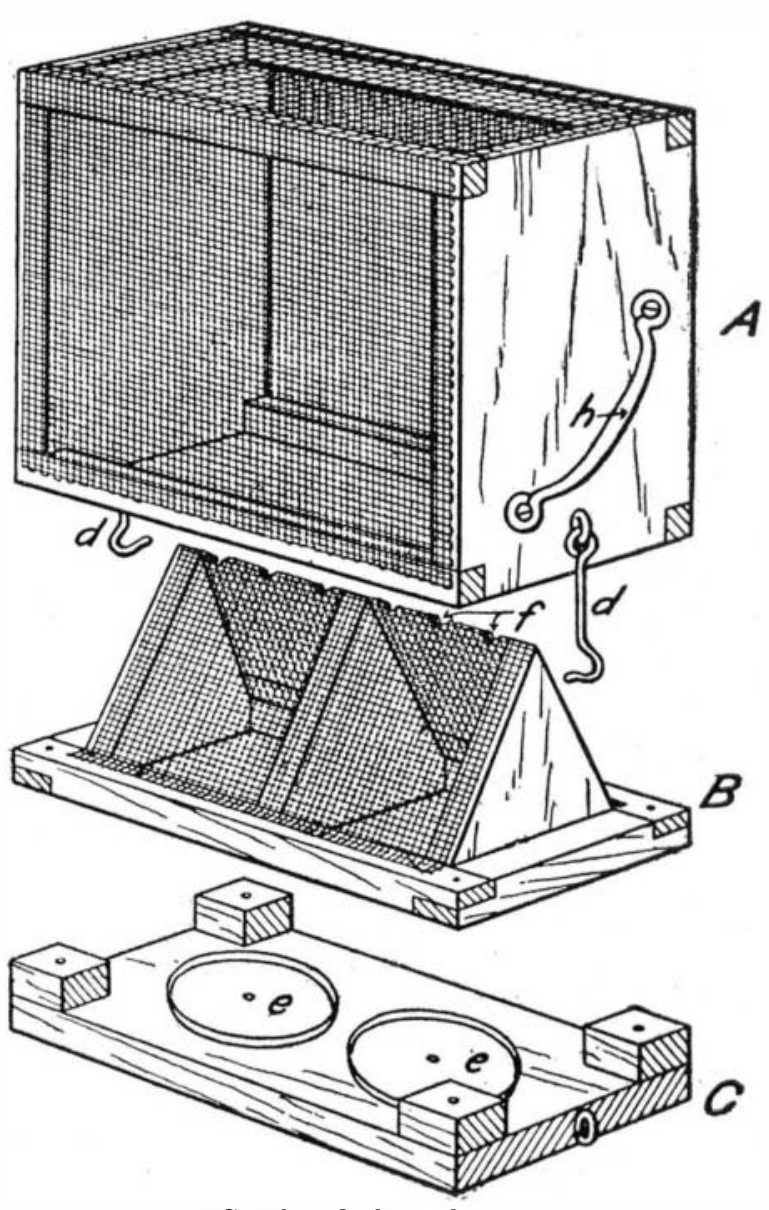

Sectional view of trap.

it is being used most successfully in the work of home sanitation.

"These traps can be made any size to suit," says the department's agent, "but the most popular size in my

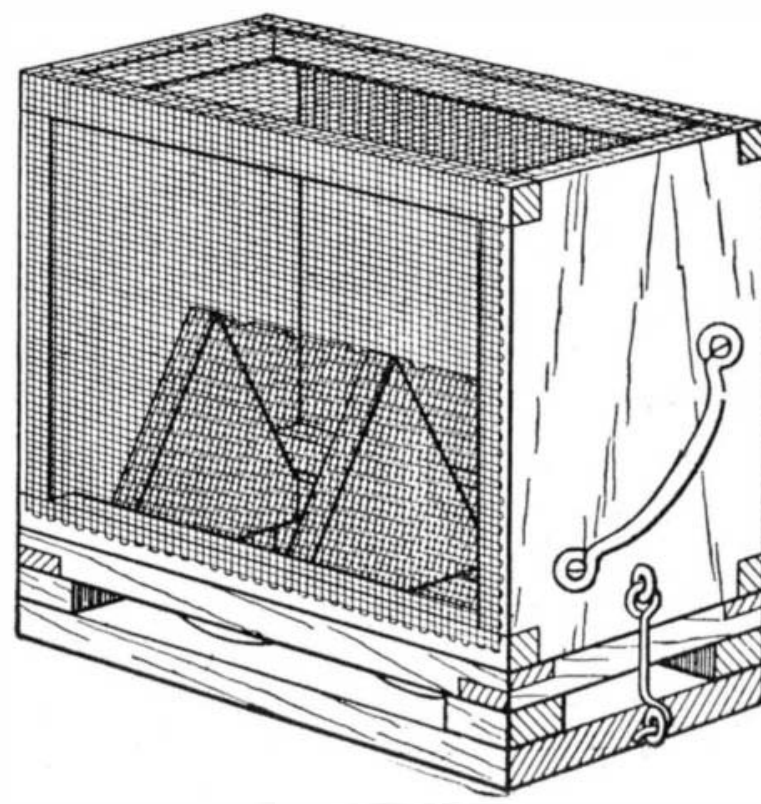

Trap ready for use.

work is 16 inches long, 12 inches high, and 8 inches wide. I also make some as large as 24 inches long, 18 inches high, and 12 inches wide. The material for these traps costs from 10 to 20 cents, and can be put together by anyone handy with tools in a short while." The trap may be baited with sour milk, a piece of banana, a fruit skin, or similar substance. This should be removed at night or it will attract ants. At the same time, the flies which have been caught should be killed by pouring hot water over the trap or leavin immersed in water until the flies are died.

The fly trap is made in three distinct parts $A, B$, and $C$, that may be detached from one another by unfastening the hooks $d$ that hold them together at either end. The trap is unhooked in order to place the bait on the bottom section $c$. The bait is placed on two pieces of tin $e$ tacked to this bottom section. In the sample trap that was forwarded to the department these pieces of tin were circular tops of paint or milk cans, which can be removed by prying up with a knife. The middle section $B$ of the trap consists of a screencovered frame that resembles a small gable roof through which there are six holes $f$ to let the flies into the uppermost section $A$, which is merely the screened-in cage that is set down over the other two parts $B$ and $C$.

The handle $h$, of course, is unnecessary, but will be found convenient when the trap is immersed in water to kill the flies.

The department's agents, in their demonstration visits to the different farms, take the fly traps with them. They interest the housewife in them, and set one up in her home to show what it can accomplish. The results of the demonstrations have been so successful that almost every farmel who sees the trap wants one, and many of them have been putting traps together in accordance with the instructions of the agent.

The traps have done much to arouse the interest of the rural population of South Carolina in fly extermination, the necessity for which is not always felt as strongly on the farm as it is in the crowded city, where these vermin make themselves more obnoxious.

WE wish to call attention to the fact that we are in a position to render competent services in every branch of patent or trade-mark work. Our staff is composed of mechanical, electrical and chemical experts, thoroughly trained to prepare and prosecute all patent ap. plications, irrespective of the complex nature of the subject matter involved, or of the specialized, technical, or scientific knowledge required therefor.

We also have associates throughout the world, who assist in the prosecution of patent and trade-mark applications fled in all countries foreign to the United States. Patent Solicitors,

361 Broadway,

Branch Office:

5 F Street, $\mathrm{W}$

Washington, D. C.

\section{SCIENTIFIC AMERICAN SUPPLEMENT \\ Founded 1876}

NEW YORK, SATURDAY, OCTOBER 10, 1914

Published weekly by Munn \& Company, Incorporated

Charles Allen Munn, President; Frederick Converse Beach Secretary; Orson D. Munn, Treasure
all at 361 Broadway. New York

Entered at Post Office of New York, N. Y., as Second Class Matte Copyright 1914 by Munn \& Co., In

The Scientific American Publications Scientific American Supplement (established 1876) per year $\$ 5.00$

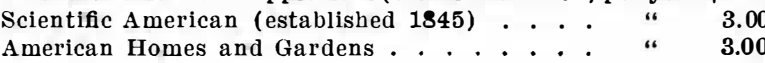

The combined subscription rates and rates to foreign countries,

including Canada, will be furnished upon application

Tunn \& Co., Inc, 361 Broadway, New York

The purpose of the Supplement is to publish the more important announcements of distin guished technologists, to digest significant articles that appear in European publications, and altogether to reflect the most advanced thought in science and industry throughout the world.

\section{Table of Contents}

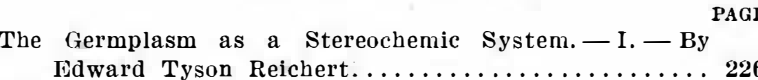

The Parseval Airship Used by the German Army.-By Carl Dienstbach.-Illustrated .................. 228

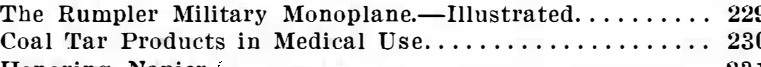
onoring Napier $\ldots \ldots \ldots \ldots \ldots \ldots \ldots \ldots \ldots \ldots . \ldots 23$

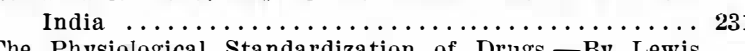
Physiological Standardization of Drusg. - By Lewis
Davis, M.S.-Lllustrated ..................... 282 ; ; Lecture for Beginners.-By Charles H. Bromley. Calculation of the Condenser Dielectric Constant.-By 234 Kenneth Richardson ........................ 23 The Cost of Building the Panama Canal............ 23

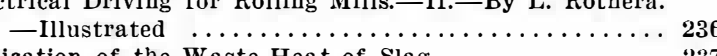

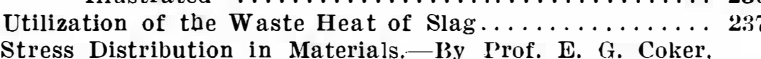

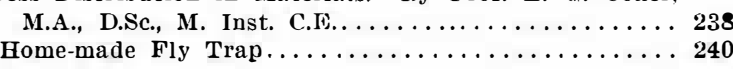

